\title{
La gubernamentalidad y el dispositivo científico-político del riesgo: la teoría de los factores de riesgo psicosocial
}

\author{
Governmentality and the scientific-political dispositive of risk: the theory of psychosocial risk \\ David Martínez (david.martinez@uss.cl) Escuela de Trabajo Social, Universidad San Sebastián (Santiago, \\ Chile) ORCID: 0000-0001-5233-5775
}

Wilson Muñoz (wilsonsocio@gmail.com) Facultad de Educación y Humanidades, Universidad de Tarapacá (Arica, Chile) ORCID: 0000-0002-6496-500X

\begin{abstract}
This article examines the conditions that underpin risk factor theory. It starts discussing the neoliberal governmentality as a political paradigm (Foucault). In this paradigm, one of the chief value is individual responsibility, where politics has as its task to normalise those that cannot adapt to that value. This normalisation is embedded in the practices which pertain public policies and social intervention. To shed light on these practices, the paper develops Foucault's concept of dispositive. In this context, one of the main dispositive is risk factor theory. It arises from the concept of risk, and by means of an historical reconstruction of this notion, we show that this theory does not reconstruct a natural condition of some people; rather, it is a contingent selection used in the context of the neoliberal governmentality.
\end{abstract}

Key words: neoliberal, governmentality, public, policy, social, intervention, dispositive, risk, factor, theory.

\section{Resumen}

Este artículo examina las condiciones para la emergencia la teoría de factores de riesgo. Comienza dando cuenta de la gubernamentalidad neoliberal como paradigma político (Foucault), donde uno de sus valores centrales es la responsabilidad individual y la política tiene como función normalizar a los segmentos que no se adaptan a este valor. Esta normalización se concretiza en las prácticas asociadas a las políticas públicas y la intervención social. Para iluminar estas prácticas utilizamos el concepto de dispositivo propuesto por Foucault. En este contexto, uno de los dispositivos relevantes es la teoría de factores de riesgo. Ella surge del concepto de riesgo, y a través de una reconstrucción histórica de esta noción, mostramos que ésta no reconstruye una condición natural de algunas personas, sino que es una distinción contingente utilizada en el contexto de la gubernamentalidad neoliberal.

Palabras claves: gubernamentalidad, neoliberal, políticas, sociales, intervención, dispositivo, riesgo, teoría, factores. 


\section{Introducción}

En este artículo se estudian las relaciones entre las lógicas de la política y la ciencia presentes en la teoría de factores de riesgo psicosocial (TFRP en adelante), tratando a esta última como un dispositivo (Foucault). Consideramos que la TFRP es una forma privilegiada de articular políticas sociales e intervenciones sociales, siendo relevante indagar las formas en que dicha teoría relaciona saberes y prácticas. Suponemos que las políticas sociales y la intervención social no son entidades abstractas, sino que se juegan en los intersticios donde el poder del Estado se vincula con la población. En contextos donde existe la figura de un Estado subsidiario, como en Chile, las políticas sociales y la intervención social se focalizan en aquellos grupos de la ciudadanía que viven en situación de pobreza, de exclusión social, y que sufren la vulneración de sus derechos. La condición de vulnerabilidad que afecta a estas personas hace necesario saber concretamente qué es lo que hacen las políticas sociales y la intervención. En caso contrario, es probable que la misma política vulnere nuevamente a individuos ya vulnerados por estructuras de desigualdad ancladas en la sociedad, reproduciendo esta situación al infinito. Violencia contra la infancia, contra adultos mayores, de género, son solo algunos ejemplos que muestran que la violencia es estructural y no es esporádica ni marginal.

La delicada situación de la infancia en Chile evidencia que se trata de un caso claro de doble vulneración. En Chile se produce la paradoja que aquellas instituciones que deberían detener y reparar las vulneraciones de derechos sufridas por niñas y niños, no lo hacen, pues sus condiciones y prácticas muchas veces replican la vulneración. En este contexto, el trabajo social es un actor clave y tiene bastante que aportar desde el momento en que su principal objeto es la intervención social, tal y como señala Saavedra en La paradoja de la negación en la intervención social. Además, esta disciplina es relevante en tanto ha dado importantes pasos para desmontar dichas estructuras de vulneración desde la mirada crítica, como la propuesta elaborada por Teresa Matus y Karen Healy.

El objetivo del artículo es mostrar cómo una teoría que parece ser solo una construcción científica, es deudora de categorías históricas-normativas vinculadas al orden político de la sociedad contemporánea y es parte clave de lo que Foucault denomina como gubernamentalidad neoliberal. Con la finalidad de acotar el campo de observación, centramos nuestro análisis en la TFRP y su estudio de la infracción de ley juvenil. De esta forma se examinan las superficies que permiten la emergencia de la TFRP como un dispositivo. Entre estas se debe destacar: la concepción de responsabilidad individual del sujeto, la noción de normalización, y el concepto de riesgo que solo surge en el contexto de la sociedad moderna. Este complejo respondería a la gubernamentalidad neoliberal, donde la fuente del riesgo es el individuo y sus decisiones. En este escenario, la política y el trabajo social buscarán resolver este asunto contribuyendo a la reconducción de los individuos hacia la norma.

Nuestro análisis utiliza como presupuesto la tesis de Michel Foucault que sostiene la existencia de un vínculo indisoluble entre el poder y saber en la sociedad contemporánea. En este contexto, los dispositivos juegan un rol clave, pues son articulaciones nacidas del cruce entre el saber científico y el poder político, los cuales pueden producir tanto conceptos como prácticas sociales. Así, los dispositivos científicos de reconstrucción de la realidad social no son ajenos a las concepciones normativas y políticas específicos de su tiempo histórico. En nuestro análisis seguimos la estrategia de trabajo propuesta por Foucault para analizar las formaciones y prácticas discursivas propuestas en La arqueología del saber y en Las palabras y las cosas. Concretamente mostraremos que la TFRP es un dispositivo que no reconstruye categorías 
naturales del mundo, ni cualidades intrínsecas de los individuos, sino que emerge históricamente y construye sus objetos a partir de una episteme particular.

\section{Gubernamentalidad neoliberal y políticas sociales}

Según Foucault, en Naissance de la biopolitique, la gubernamentalidad neoliberal juega un rol fundamental en la conformación de los dispositivos científicos y políticos que generan a las políticas sociales. Entre las categorías centrales de esta forma de gobierno destacan el concepto de riesgo, el cual privilegia la norma de la autorregulación individual por sobre otros valores como la justicia, la equidad y la solidaridad. De esta forma, es el individuo el principal responsable de su estilo de vida, auto-cuidado y bienestar. Y si la persona no responde adecuadamente a los estándares normativos, el Estado, las políticas y las leyes operarán a través de técnicas disciplinarias más o menos coercitivas. En el caso particular de la juventud, la infracción de las leyes genera una reconstrucción discursiva que permite la instalación de prácticas de observación y control donde juegan un rol fundamental las políticas sociales. En este orden del discurso, donde se cruzan tanto los saberes científicos como las lógicas políticas, la intervención social juega un rol protagónico.

De ahí que sea acertado sostener que una de las funciones latentes de la política ha consistido en aportar al control de las poblaciones a través de "prácticas de normalización" (Chambon et al. 1999:67). Siguiendo la reflexión en la línea inaugurada por Foucault, Laura Epstein discute dicho concepto y señala que "el sentido de normalizar consiste en hacer conforme a una norma o estándar, a través de la transformación de elementos en una persona o situación" (Epstein 1999:9). Es así como muchas políticas sociales y programas se van constituyendo como un aparato que refuerza la ideología de la responsabilidad individual, donde las personas tienen la obligación normativa de desarrollar de la forma más lograda posible sus proyectos de vida dentro de su marco de posibilidades. Lógicamente, dicho esfuerzo individual no puede ir contra las leyes del Estado de Derecho. Los jóvenes que fallecieron en un incendio en la cárcel de San Miguel de Santiago en 2010 (muchos de ellos simplemente por trabajar en el comercio informal), son un ejemplo macabro de esa normatividad que exige responsabilidad individual, pero sin transgredir las normas del Estado de Derecho. En consecuencia, en el contexto del neoliberalismo, los problemas sociales se explican fundamentalmente por las incapacidades del individuo para adaptarse a las normas. De ahí la narrativa de la meritocracia y su premisa: la asignación de recursos en la sociedad responde a las capacidades de los individuos, y los desfavorecidos lo son porque son menos competentes. Así, la estructura sistemático-histórica de vulneraciones y desigualdades queda simplemente invisibilizada.

En el caso de la infracción de ley por parte de jóvenes, el marco legal es la Ley 20.084 y se constituye fundamentalmente como un orden normativo de responsabilidad penal adolescente. Este título ya supone ciertas decisiones semánticas, pues parte de la idea que los adolescentes son ciudadanos, y por ende son titulares de derechos; pero a la vez tienen obligaciones con el resto de la sociedad y el Estado. Finalmente son ellos los principales responsables de las acciones y decisiones que toman a lo largo de su vida. Probablemente también sus madres y padres y los grupos de pares, pero no el Estado ni menos la estructura social. Ahora bien, en tanto titulares de derechos, para estos jóvenes se aplican formalmente las normas constitucionales y el ordenamiento internacional (por ejemplo, la Convención Internacional de Derechos del Niño), pero la realidad cotidiana nos muestra que nadie sabe muy bien cómo garantizar que esos derechos efectivamente se implementen a través de prácticas e instituciones. 
En cambio, en el ámbito de las responsabilidades, lo que constituye una transgresión es conocido. Y en caso de que no se conozca, los juzgados, las policías y en última instancia el sistema punitivo hace que dicho marco normativo se respete. Si consideramos este doble aspecto de la ciudadanía, veremos que el trabajo social tiene innumerables y excelentes ejemplos para observar la paradoja entre responsabilidades y derechos. En el caso de la infracción de ley, la mayor parte de las veces las biografías de las personas que transgreden las normas se caracterizan por trayectorias de vulneraciones de derechos desde el nacimiento. En un país como Chile, donde el gasto social aún es relativamente bajo y existen fuertes niveles de desigualdad, pareciera que siempre falta institucionalidad para responder a esas vulneraciones, deteniéndolas y reparándolas. Sin embargo, cuando las víctimas se convierten en victimarios o simples transgresores de normas menores que no ponen en riesgo a la sociedad en su conjunto (como en el caso del comercio informal), la institucionalidad del Estado de derecho hace sentir toda su fuerza y los cuerpos ya marcados desde el origen se marcan nuevamente. $Y$ en los casos más extremos, esas marcas pueden transformarse en muerte.

Una vez discutida la relación entre la gubernamentalidad neoliberal, las políticas sociales, y un fenómeno concreto donde se ejerce el poder político como la infracción de ley por parte de jóvenes, es necesario mostrar qué hacen específicamente las políticas sociales. En este contexto, consideramos que el trabajo social puede realizar una contribución clave, pues su objeto coincide en gran medida con el de las políticas sociales, a saber, la intervención social.

\section{La construcción del objeto en las políticas sociales y el trabajo social}

Es importante comenzar señalando que las políticas sociales y el trabajo social no se producen con individuos en cuanto tales. Tal como señala Teresa Matus, "nadie llega «en su condición de persona natural» a solicitar los servicios de un trabajador social, sino que emerge al interior de una categoría analítica determinada" (Matus 2003:55). Por tanto, el núcleo de la intervención es una intersección, un cruce entre los sujetos y el fenómeno social que los convoca. Consecuentemente, "si la categorización social se realiza en términos estigmatizadores, esos sujetos llevarán esa marca en forma persistente. De allí que estudiar los modelos de intervención social que se realicen y sus formas enunciativas, resulta clave en el logro de mayores oportunidades para el desarrollo y el fortalecimiento de la ciudadanía" (Matus 2003:55).

Asimismo, la intervención es "una forma de ver que funda un hacer peculiar" (Matus 2003:56) y adicionalmente conlleva una determinada forma de relación entre los sujetos, en tanto los implicados en la intervención no existen fuera de la actividad histórica del trabajo social. De esta forma, el punto de partida no está dentro de los usuarios, sino dentro de la intervención social misma. Tal como señala Chambon, "los usuarios y los trabajadores sociales son definidos al interior de arreglos culturales e institucionales y en sistemas de poder" (Chambon 1999:59). De esta forma, lo que se pone en juego en la intervención viene definido por estructuras de significados que se traducen en prácticas concretas.

Por esto los dispositivos de intervención nunca se pueden entender como estrategias que realizan observaciones hacia el mundo tal como es, a través de las cuales se captaría la realidad en sí de las personas y sus circunstancias. Por el contrario, es necesario reconocer que siempre se opera desde un marco conceptual y epistémico particular. Si no se reconoce el hecho de que se está operando desde una reconstrucción, las personas se vuelven una identidad con las categorías sociales que las identifican y no se reconoce que operaría una observación particular: una elaboración del sujeto-objeto desde las políticas 
sociales y el trabajo social. Nadie se autodefine a priori como «menor en situación irregular» o «joven en riesgo». Por ello, existe un sistema enunciativo frente al que será preciso localizar las superficies de su emergencia. Es así como la tarea consiste en explicitar los dispositivos de orden que identifican ciertas conductas como desviación, anomalía o conducta de riesgo.

Para Foucault, en La arqueología del saber, estos planos de emergencia no son las mismas para las distintas sociedades, épocas, y formas de discurso. En el proceso de construcción del sujeto en el contexto de la gubernamentalidad neoliberal, la ciencia y la política se han apoyado cada vez más en prácticas de cosificación de las personas y sus cuerpos, lo que ha devenido en una extensión del control social. Cuando se olvida que en las políticas sociales y la intervención social se construye un nombre o una categoría, se puede caer en esa lógica cosificadora. Consecuentemente una de las tareas de la política consistirá en conducir a las poblaciones problemáticas hacia la norma o estándar esperado. Ese es uno de los límites de toda intervención social: olvidar que, en su conformación y aplicación, hay un momento de selección y de observación que funda un hacer particular. Esa omisión consiste en suponer que la observación es una representación de una evidencia exterior. Consideramos que una manera de contribuir a problematizar y contextualizar este fenómeno es considerando a estas políticas sociales y sus fundamentos teóricos como dispositivos. Para avanzar en esta línea, en la siguiente sección mostraremos en qué consiste este concepto según Foucault.

\section{El concepto de dispositivo}

La política pública y la intervención social ponen en movimiento dispositivos que son formas de ver y de reconstrucción de los sujetos que devienen en prácticas particulares. Aquí debemos recordar que el concepto de dispositivo ha sido ampliamente tratado por autores como Deleuze, Agamben y Hardt y Negri. Todos ellos son herederos directos de la reflexión de Michel Foucault sobre este concepto, y más allá de sus diferencias y aportes particulares a la discusión, en general mantienen la intuición original del filósofo de Poitiers: el dispositivo es una red que no puede ser entendida meramente como un discurso, una cosa, o una manera de ser (sujeto), sino como la articulación que se establece entre éstos. No podemos detenernos aquí a discutir las diferencias y aportes de cada uno de estos autores, asunto que superaría los límites de nuestro artículo. Además, consideramos que la propuesta de Foucault es lo suficientemente compleja y potente para iluminar nuestro problema de estudio particular, de manera que abordaremos las especificidades de este concepto atendiendo únicamente a su propuesta teórica.

Para Foucault, los dispositivos son articulaciones discursivas que emanan del cruce entre el saber científico y el poder político. Esta noción puede ser entendida en primer lugar como una red de conceptos. Consistiría en una articulación compleja que posee distintos niveles, y que a su vez no es homogénea, pues en ella se entrecruzan una pluralidad de elementos. Además, el dispositivo no solo produce un entramado conceptual, sino que también produce un conjunto de prácticas propias del orden del discurso. De esta forma, los dispositivos en políticas sociales y en la intervención social son entramados conceptuales o semánticos que producen formas de hacer, prácticas y estructuras. Sin embargo, los dispositivos son también herramientas teóricas y metodológicas, pues permiten el trabajo de deconstrucción, que entre otras cosas hace saltar el mito de lo dado y la cosificación de los discursos y las prácticas. En otras palabras, la reconstrucción o deconstrucción de los dispositivos permite desmontar las estructuras discursivas que establecen formas de poder. Solo como ejemplo, piénsese en el sistema patriarcal estudiado por Guzmán y Pérez en La teoría de género y su principio de demarcación científica) o en el racismo que es investigado 
por Alvarado y Fernández en Una narración fundacional para una antropología filosófica chilena: Raza Chilena de Nicolás Palacios.

El dispositivo además posibilita la constitución de series y agrupamientos que se articulan a partir de cuatro esquemas o principios que permiten constituir un sistema de "gramática general" (Foucault 2006:86): la atribución, la articulación, la designación y la derivación. Estas nociones, a través de sus relaciones, constituyen los órdenes discursivos. Gracias a cada una de estas operaciones se generan las condiciones de emergencia de los diversos objetos particulares del conocimiento, las estructuras y las prácticas que median las relaciones entre la política y los sujetos nombrados en esas definiciones. Ahora bien, tres son las características fundamentales de la mencionada gramática general. En primer lugar, constituye un dominio de validez, definiendo qué criterios se pueden discutir en cuanto a la verdad o el error de una proposición. En segundo lugar, constituye también un dominio de normatividad, donde "según qué criterios se excluyen ciertos enunciados como no pertinentes para el discurso, o como inesenciales y marginales, o como no científicos" (Foucault 2007:99). En tercer lugar, definen un dominio de actualidad "que comprende las soluciones logradas, que define los problemas presentes, que sitúa los conceptos" (Foucault 2007:99). Estas tres dimensiones definen un único orden: el orden del discurso.

Como ya se ha señalado, cada una de estas dimensiones define a su vez ciertas prácticas discursivas. En Vigilar y Castigar, por ejemplo, Foucault comienza describiendo los suplicios a los que era sometido un condenado en el siglo XVIII. Quien no conoce la formación de los conceptos de tal régimen punitivo, no puede entender su lógica. Ellos encuentran su correspondencia en las tres características anteriormente nombradas del orden del discurso: la dimensión de la validez, de la normatividad y de la actualidad. En el caso del sistema penal en el siglo XVIII: los criterios de verdad que justifican dicho régimen punitivo; los principios que son excluidos; y finalmente las formas de castigo que se actualizan en un orden determinado.

Las estrategias son un componente adicional en la constitución de un dispositivo. Ellas son configuraciones disciplinares que constituyen una lógica que abre posibilidades de observación, y por lo mismo, definen ciertos elementos y su organización posible. En este punto, Foucault indicará: "Discursos como la economía, la medicina, la gramática, la ciencia de los seres vivos, dan lugar a ciertas organizaciones de conceptos, a ciertos reagrupamientos de objetos, a ciertos tipos de enunciación, que forman según su grado de coherencia, de rigor y de estabilidad, temas de teorías" (Foucault 2007:86).

Por lo tanto, la forma de enunciación de un discurso implica la manera en que se configuran los sistemas conceptuales y de elección de teorías. Y esto a su vez determina las configuraciones de las disciplinas, que desde sus diversos puntos de observación elaboran sus objetos y sus prácticas.

El concepto de dispositivo también se puede comprender considerando lo que quiere decir Foucault con los límites del mundo. Estos se definen como el espacio donde los seres humanos habitan. Sin embargo, eso no significa que tengan que ver con una delimitación geográfica o territorial. De lo que se trata es de una estructura compuesta por creencias sobre el mundo que abre el campo de lo visible, lo pensable y lo comunicable. Para Foucault son las formaciones discursivas las que articulan el dispositivo a partir del cual el mundo se ilumina. No solo el mundo físico y natural, sino también el mundo humano de los significados y las relaciones sociales. El filósofo francés recurre a la figura de Don Quijote para ejemplificar cómo el dispositivo es capaz de trazar los límites del mundo. El personaje de Cervantes está cautivado y a la vez prisionero de una cierta narrativa: las novelas de caballería. De esa forma, habita en ese dispositivo y en 
esos límites. Y así Don Quijote: "Es el héroe de lo Mismo. Así como de su estrecha provincia, no logra alejarse de la planicie familiar que se extiende en torno a lo Análogo. La recorre indefinidamente, sin traspasar jamás las claras fronteras de la diferencia, ni reunirse con el corazón de la identidad [...] Las novelas de caballería escribieron de una vez por todas la prescripción de su aventura" (Foucault 2006:53).

Por ello, según Las Palabras y las Cosas, la actitud de Don Quijote es la perfecta conducta del encierro, de la repetición, de la unicidad frente a las cosas y los procesos del mundo. En otras palabras, la incorporación de toda diferencia del mundo en el marco de una estructura invariable.

El lenguaje supone una representación que se vuelve posible por los límites impuestos a través del orden discursivo y es así como la posibilidad del habla supone una marca, una diferencia. Y esto delimita un espacio donde se define lo pensable y lo decible. Aquí el error se encuentra en el olvido de ese momento de elección de la designación y de la naturaleza metafórica del lenguaje, pues constituye una equivocación obviar que existe un entramado desde donde surge lo que se nombra. Cuando se reconoce que detrás de todo objeto de conocimiento existe un dispositivo, es posible articular un discurso de oposición, de crítica y eventualmente de transformación; pero cuando eso no existe, pareciera ser que el orden del discurso es el orden natural del mundo, como lo era el mundo caballeresco para Don Quijote. En ese caso se está pensando dentro de los límites de la ontología, y tanto los significados como los humanos se constituyen en esencias. Consecuentemente, las relaciones sociales se naturalizan y, por ejemplo, ser pobre y joven deviene casi naturalmente en ser persona problemática y en riesgo.

Una vez realizadas estas reflexiones epistemológicas, en la siguiente sección discutimos cómo el riesgo aparece como una condición de posibilidad de un régimen de enunciación particular. Luego mostraremos concretamente que el riesgo es la categoría central que permite el nacimiento de un dispositivo de las políticas públicas y la intervención social que aborda el problema de la infracción de ley juvenil: la teoría de factores de riesgo. Teniendo en consideración la genealogía del concepto de riesgo, se puede intuir que esta teoría no es simplemente el reflejo o la representación de ciertas características intrínsecas a algunos seres humanos. Esta es la tarea de reconstrucción que ofrece propuesta por Foucault para desmontar los modos de cosificación del ser humano.

\section{Orígenes históricos y conceptualización sociológica del riesgo}

En términos históricos, el origen del riesgo se puede rastrear en los siglos XVII y XVIII en el contexto del juego y las apuestas. Según Mary Douglas, en ese marco, se fue desarrollando un análisis especializado de las probabilidades de ganancia y perdida asociadas a dichas actividades. A su vez, en estos siglos el análisis del riesgo tenía importantes usos en la navegación, y se entendía como una categoría neutral que tomaba en cuenta las probabilidades de sufrir accidentes. Por ello, el riesgo se asociaba a la relación de los individuos con la naturaleza misma y el énfasis del riesgo se encontraba en el medio ambiente.

En la economía liberal del siglo XIX el foco se modifica y el riesgo ya no se observa desde el punto de vista de la relación entre los sujetos y las fuerzas inmanejables de la naturaleza. Más bien, el riesgo se reconstruye en relación con el actor económico racional y sus selecciones. Según este modelo, la tendencia natural del homo economicus sería el rechazo del riesgo, pues sus acciones deberían orientarse a la maximización de beneficios y a evitar todo tipo de amenaza o perdida asociada a elecciones irracionales. Sin embargo, el actor económico puede incurrir en riesgos siempre y cuando exista el incentivo de un 
beneficio asociado a tomar ciertas decisiones. Esto se observa sobre todo en la figura de los empresarios y "el cálculo del riesgo y los posibles beneficios asociados a una inversión" (Douglas 1992:23).

En síntesis, se puede ver que (al menos desde el siglo XVII) el análisis de las probabilidades y del riesgo se ha convertido en uno de los pilares articuladores de muchas decisiones humanas. Las ciencias de las probabilidades y la economía se instalan entonces como fundamentos donde la sociedad encuentra su orden. Además, la incorporación del riesgo constituye una categoría que transforma la naturaleza de la evidencia, del saber y también de la política y el gobierno. Desde esta nueva perspectiva de observación de lo social, todo proceso podría fallar, y es así como el cálculo del riesgo se integra profundamente en distintos ámbitos, operando como una base para los procesos de toma de decisiones. Tal como indica Douglas, el concepto de riesgo se ha instalado en la política, en tanto el pensamiento probabilístico se ha generalizado en la industria, las ciencias modernas y la filosofía.

El concepto de riesgo también ocupa un lugar protagónico en la sociología contemporánea. Una breve síntesis de las reflexiones generadas por Beck (La sociedad del riesgo) y Luhmann (Sociología del riesgo) nos darán algunas pistas sobre la concepción actual que posee esta disciplina al respecto. Para Beck, la principal característica de la sociedad moderna es constituirse como una sociedad del riesgo. Esto implica un cambio en la matriz cultural, pues en esta sociedad el pilar normativo lo constituye la seguridad y el ideal esperado es la eliminación y control del riesgo. Por su parte, Luhmann distingue entre riesgo y peligro. El primero se explica por las consecuencias de la propia selección del sistema y el segundo es atributo del entorno. Por ende, el riesgo tiene que ver con posibles consecuencias no esperadas de la acción. En virtud de esto, en la sociedad contemporánea adquieren un rol central la ciencia y los expertos que buscan controlar y minimizar dichos riesgos. De esta forma, el concepto de riesgo implica su gestión o gobierno a través de sistemas especializados. En el caso de los problemas sociales, que es lo que nos interesa en este artículo, el riesgo exige respuestas desde las políticas y desde la intervención social. Ambos autores sostienen que la sociedad contemporánea se caracteriza por un alto sentido del riesgo, donde cualquier tipo de daño o consecuencia adversa se origina por las decisiones humanas. Por lo mismo, la evaluación del riesgo, su manejo y minimización se han convertido en elementos esenciales de la política y las ciencias. Es así como, a través de un entramado de instituciones y prácticas la sociedad busca responder a un problema auto-generado por medio de la planificación y la intervención.

Considerando estos antecedentes podríamos reducir nuestro foco y argumentar que la noción de riesgo se ha vuelto una categoría esencial de la política y la normatividad. El riesgo es el riesgo de que el individuo (o cualquier organización social desde una asociación a un Estado) elija equivocadamente. Por ello, la categoría es eminentemente normativa, pues tiene que ver con la libertad humana y su ejercicio más o menos responsable. En el caso particular de la juventud, la atribución es que las personas que se encuentran en esta etapa están en un periodo de transición asociado con altos niveles de riesgo. Por ende, es un periodo que está abierto a grandes ganancias asociadas a elecciones positivas, pero que también puede resultar en lo contrario. Al estar en una etapa del ciclo vital caracterizada por muchas posibilidades de acción, algunos jóvenes pueden tomar riesgos que podrían desencadenar en resultados negativos para su propio bien y el de los demás. Y efectivamente es posible mostrar que, desde las políticas sociales, la juventud es considerada una etapa de la vida de las personas que se asocia al riesgo: "La etapa de la juventud es particularmente crucial en la definición de la identidad de la persona como ciudadano. Es una época de experimentación y riesgo que requiere de atención social, familiar y estatal, en la que es importante conocer y diseñar políticas públicas que partan de la idea de transitoriedad, de construcción de autoestima [...] y de sentido de pertenencia a redes sociales" (Solís 2007:145). 
En resumen, el concepto de riesgo es una categoría eminentemente histórica y supone una serie de condiciones particulares para su emergencia. Como veremos a continuación, olvidar este carácter implica opacar la condición de dispositivo de la teoría de factores de riesgo, lo que conlleva a su naturalización y a suponer que el riesgo es una propiedad intrínseca de ciertos tipos de individuos: como los jóvenes en situación de pobreza material, por ejemplo. A la vez, en esta operación se invisibilizan una serie de prácticas e instituciones asociadas al poder político y que poseen una materialidad concreta a través del ejercicio de las políticas sociales.

\section{La teoría de factores de riesgo}

El enfoque de factores de riesgo emerge de la perspectiva psicosocial, la cual "permite o facilita un modo de elaborar el conjunto de problemas de una situación global o de las cuestiones que afectan la calidad de vida de una persona, generando al mismo tiempo una posibilidad diferente y más eficaz de actuar los modos de intervención" (Asún 1994:353). Los elementos que permiten avanzar en la definición de un problema psicosocial son el contexto, entendido como el "entorno ambiental y social en el cual se encuentra la persona y con el cual se relaciona", el bienestar psicosocial, que se puede definir como "la satisfacción de las necesidades psicológicas, afectivas, sociales" (Asún 1994:353) y los factores de riesgo.

Según Hein y Barrientos (2004), los factores de riesgo son condiciones sociales, comunitarias e individuales o personales que obstaculizan el logro del bienestar e incrementan las probabilidades que las personas desarrollen problemas emocionales, conductuales y de salud. Los factores de riesgo "conducen a actitudes peligrosas e inhiben el desarrollo saludable" (Bogenschneider 1996:127). Y un comportamiento de riesgo es "cualquier tipo de conducta que impide el desarrollo del individuo y que puede comprometer sus competencias, sus habilidades y la adquisición de roles socialmente aprobados" (Mancini y Huebner 2004:648).

Respecto a los aportes de la perspectiva de factores riesgo se pueden destacar: la generación de un lenguaje común entre los distintos actores involucrados en las políticas públicas; su aplicabilidad "a diversos fenómenos sociales, como el envejecimiento, infancia, salud, justicia juvenil, salud mental, uso de drogas, entre otros" (Fraser 2004:213) y su aporte potencial "a la formulación de políticas públicas en la medida en que sea capaz de detectar variables que incrementen (factores de riesgo) o disminuyen (factores protectores) la probabilidad de un determinado comportamiento de riesgo" (Hein y Barrientos 2004:7). En el mismo sentido, "si conocemos los factores que llevan a estas conductas arriesgadas, estaremos en mejor posición para prevenir las consecuencias de éstas e, incluso, para tratarlas mejor cuando se presenten" (Florenzano 1997:1).

En el caso de la infracción de ley en adolescentes, los diversos factores y/o variables ayudan a observar las principales causas que devienen en la transgresión de la norma. Entre dichos factores se pueden destacar variables asociadas a los individuos, el grupo de pares, la familia y la dimensión social y comunitaria (Munizaga 2009:7). Lo importante aquí es que en el marco de esta teoría se articulan muchas de las intervenciones e investigaciones que surgen al alero de instituciones como el Servicio Nacional de Menores (SENAME), donde se reconoce que: "La actual tendencia a trabajar con teorías orientadoras para comprender la infracción de ley en los adolescentes, acarrea la necesidad de identificar aquellos factores claves, alrededor de los cuales se nuclean todos los demás. Hay distintos factores que, de acuerdo con la investigación comparada, influyen en la formación de diversos tipos de compromiso delictivo en los adolescentes" (SENAME 2007:9). 
Como podemos apreciar, la teoría de factores de riesgo no es solo un aparato conceptual o simplemente una posibilidad de reconstrucción teórica para la descripción de un fenómeno problemático. Más bien constituye un dispositivo de observación que resulta clave para las políticas públicas específicas y las formas de intervención social asociadas. Por ende, y como sostiene Foucault, no es solo una red conceptual, sino también una red de prácticas sociales e instituciones.

A la luz de la idea de Foucault de los límites del mundo, si la TFRP no es utilizada con la suficiente percatación epistemológica en la intervención social, su uso irreflexivo nos puede conducir a la perfecta conducta del encierro y la repetición. $Y$ en el caso del trabajo con jóvenes en conflicto con la justicia, ese encierro se puede traducir en prácticas que difícilmente pueden avanzar hacia el desarrollo de esas personas y sus potencialidades. Si el riesgo es el punto de partida, entonces es difícil ver en estos jóvenes otros recursos, proyectos, ideales, sueños. Incluso el ímpetu por el reconocimiento se opaca en su potencial de desarrollo de una identidad autónoma y lo que queda es un sujeto (objeto) con problemas con la justicia, en una situación irregular, con consumo problemático de drogas y probablemente fuera del sistema escolar.

\section{Conclusión}

En este artículo se ha mostrado que uno de los elementos que se utilizan en las políticas públicas y la intervención social, la teoría de factores de riesgo, emerge históricamente como un dispositivo donde se vinculan ciertos discursos y lógicas surgidos en la sociedad contemporánea y que devienen en prácticas específicas. Estos discursos y prácticas se insertan en un contexto global donde el paradigma de gobierno es la gubernamentalidad neoliberal (Foucault). Dicho marco político se asocia a ciertos valores y a una normatividad que deviene en gobierno de las personas y sus cuerpos, siendo uno de sus principios fundamentales la responsabilidad individual. Es aquí donde la política debe aportar a la normalización de los individuos que no se adaptan a ese tipo de valores: las poblaciones en riesgo. Pero si bien son los dispositivos, en tanto entramados de discursos y prácticas, lo que permiten realizar las políticas públicas y las intervenciones sociales, la percatación epistemológica demanda reconocer en todo dispositivo una forma de ver particular que, según Matus, funda un hacer.

Así, nuestra reflexión sostiene que una teoría específica, que muchas veces informa la configuración de las políticas sociales y la intervención social respecto a la infracción de ley por parte de jóvenes, responde no solo a criterios científicos, sino que también incorpora elementos de índole político-normativos. Este ejercicio puede resultar fructífero para no naturalizar las categorías del análisis científico social, contribuyendo así a evitar algunas de las consecuencias sociales que podría tener dicha omisión. En este escenario, nuestro aporte se enmarca en el desarrollo de la rama de la política pública y el trabajo social crítico, la cual discute las categorías y dispositivos que fundamentan sus procesos de intervención social.

Finalmente consideramos que sería interesante que otras investigaciones pudieran contrastar las lógicas de la TFRP con otros enfoques utilizados en las políticas sociales y las intervenciones, como el enfoque de derechos o de inclusión social. Nuestra evidencia nos permite lanzar una hipótesis que debe contrastarse: la TFRP es incompatible con estos enfoques mencionados; lo cual podría constituirse en un hallazgo relevante si consideramos que muchas políticas e intervenciones que incorporan la semántica del enfoque de derechos o de inclusión social, finalmente, terminan operando más bien bajo el paraguas de la TFRP, lo cual implicaría una serie de limitaciones como las que hemos analizado en este artículo. 


\section{Agradecimientos}

El presente artículo es resultado de los proyectos Fondecyt 1160976 y UTA 5720-17. Se agradece también el apoyo del Convenio Marco: "Plan de Implementación para Programa de Fortalecimiento de la Formación Inicial Docente 2017-2019" UTA-MINEDUC 1656 y del Programa Becas Chile Doctorado de CONICYT.

\section{Bibliografía}

Asún, D. 1994. Acerca del enfoque psicosocial o la superación de una visión biomédica de los fenómenos juveniles, pp. 353-358. En: INJUV. Primer Informe Nacional de Juventud. Santiago: Instituto Nacional de la Juventud.

Bogenschneider, K. 1996. An ecological risk/protective theory for building prevention programs, policies, and community capacity to support youth. Family Relations 45(2): 127-138.

http://www.jstor.org/stable/585283

Chambon, A. et. al. 1999. Reading Foucault for social work. New York: Columbia University Press.

Douglas, M. 1992. Risk and blame. New York: Routledge.

Epstein, L. 1999. The culture of social work, pp. 3-25. En: Chambon, A. et. al. Reading Foucault for social work. New York: Columbia University Press.

Florenzano, R. 1997. El adolescente y sus conductas de riesgo. Santiago: Ediciones Universidad Católica de Chile.

Florenzano, R. 1997. Conductas de riesgo y factores protectores. Santiago: Pontificia Universidad Católica de Chile.

Foucault, M. 2006. Las palabras y las cosas. Una arqueología de las ciencias humanas. Madrid: Siglo XXI.

Foucault, M. 2007. La arqueología del saber. Madrid: Siglo XXI.

Fraser, M. 2004. Intervention research in social work: recent advances and continuing challenges. Research on Social Work Practice 14(3): 210-222. https://doi.org/10.1177/1049731503262150

Hein, A. y Barrientos, G. 2004. Violencia y delincuencia juvenil: comportamientos de riesgo autorreportados y factores asociados. Santiago: Fundación Paz Ciudadana.

Mancini, J. y Huebner, A. 2004. Adolescent risk behavior patterns: effects of structured time-use, interpersonal connections, self-system characteristics, and socio-demographic influences. Child and Adolescent Social Work Journal 21(6): 647-668. https://doi.org/10.1007/s10560-004-6409-1

Matus, T. 2003. La intervención social como gramática. Hacia una semántica propositiva del trabajo social frente a los desafíos de la globalización. Revista de Trabajo Social 71: 55-71.

https://repositorio.uc.cl/handle/11534/6059 
Munizaga, A. 2009. Potencialidades del enfoque de factores de riesgo. Breve revisión de las teorías del delito. Conceptos 12: 1-14.

Solís, L. 2007. Crimen organizado en América Latina y el Caribe. Santiago: Catalonia.

SENAME. 2007. Sistema nacional de atención socioeducativo para adolescentes infractores de ley, período 2006-2010. Santiago: Servicio Nacional de Menores.

Recibido el 14 Dic 2017

Aceptado el 6 Mar 2018 